\title{
Case report: mediastinal lymphoma in cat
}

\section{Introduction}

Lymphoma is the most common feline neoplasm and constitutes more than half of hemolymphatic tumors. They can be diagnosed in cats of any age, race and sex, although Siamese may have an increased risk. The precise etiology of this pathology is in many cases unknown, however, there is a high correlation between the presentation of lymphoma and viral infections such as feline leukemia virus (ViLeF) and feline immunodeficiency virus (FIV). ${ }^{1,2}$

\section{Clinical history}

Domestic shorthaired cat, two years old, castrated male, consulted in the Hospital of the Faculty of Veterinary (Universidad de la República, Uruguay), for severe dyspnea. The anamnestic data revealed that he is an indoor cat, without access to the outside and does not live with other animals. He is feed a high-quality cat food and is not vaccinated. Is not receiving any long-term medications, and the owner have not given him anything for the dyspnea.

Clinical signs began with apathy and disorder in feeding 60 days before the consultation. In the general physical examination, he presents a bad body condition, facies of anguish and breathing open mouth. The mucous membranes were cyanotic, palpable lymph nodes without peculiarities, temperature of $38^{\circ} \mathrm{C}$, filiform pulse and respiratory rate 50 per minute.

The particular examination of the respiratory system was carried out. On inspection there were no alterations in the nasal cavity but a significant restrictive dyspnea was observed. The percussion of the thorax revealed dullness, and at the thoracic auscultation no heart or lung sounds were perceived.

The chest x-ray study of latero-lateral incidence showed an increase in the radiodensity of the cranial thoracic region with soft tissue density, suggestive of cranial mediastinal mass. The presence of pleural effusion was not ruled out.

Complete blood biochemistry routine was performed, blood count and serology for FIV (FASTest FIV LabSet, Megacor Laboratory, Austria) and ViLeF (FASTest FeLV LabSet, Megacor Laboratory, Austria). The results showed mild leukocytosis with a predominance of neutrophils and a positive ViLeF test.

Due to the severity of the condition, euthanasia was performed. The necropsy revealed a tumor that occupied the entire thoracic cavity, displacing the lungs to the dorsal and enveloping the heart. The tumor presented a multinodular appearance, firm consistency and pale pink color.

In the histopathological images, there was a homogenous population of immature lymphoid cells: small lymphocytic cells, of circular type and with little cytoplasm, with nuclei from round to elliptical and with some notches. We also observed the presence of necrosis in the tumor tissue and disorganization of the original tissues (lung and myocardium). The histopathological diagnosis was thymic lymphoma (Figure 1-3).
Volume 6 Issue 2 - 2019

\section{Decuadro Alicia, Menéndez Cecilia, Ruiz Natalie, Bartesaghi Natalia, Yamasaki Kanji, Benech Alejandro}

Departamento de Patología y Clínica de Pequeños Animales, Facultad de Veterinaria, Universidad de la República, Uruguay

Correspondence: Benech Alejandro, Departamento de Patología y Clínica de Pequeños Animales, Facultad de Veterinaria, Universidad de la República, Uruguay, Email alebenech@hotmail.com

Received: June 04, 2018 | Published: April 03, 2019

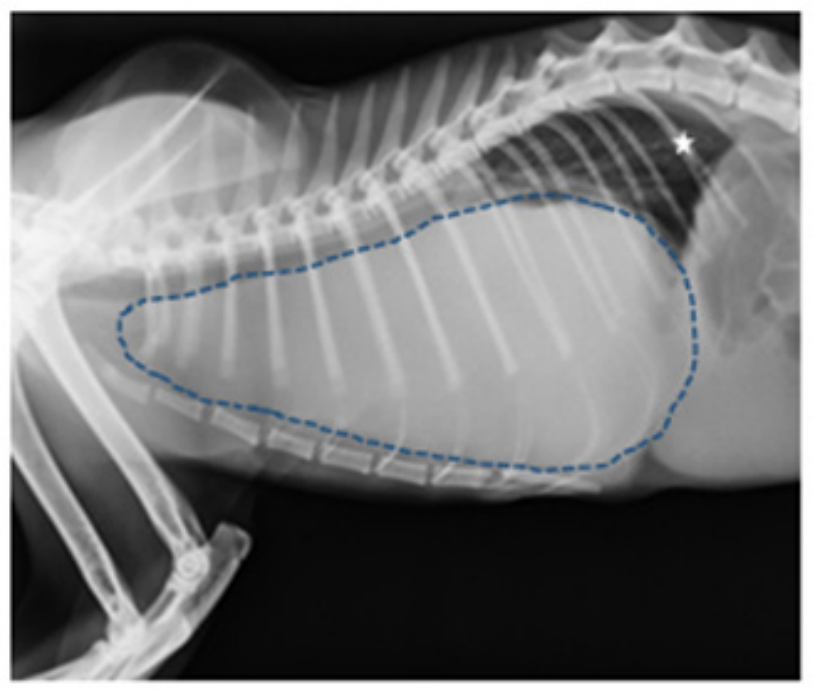

Figure I Chest radiograph, lateral incidence. There is an increase in the radiodensity of the cranial thoracic region with soft tissue density, suggestive of cranial mediastinal mass (dotted line). The lungs are compressed towards the dorsal (white star), which explains the severe dyspnea. The mass does not allow seeing the cardiac silhouette.

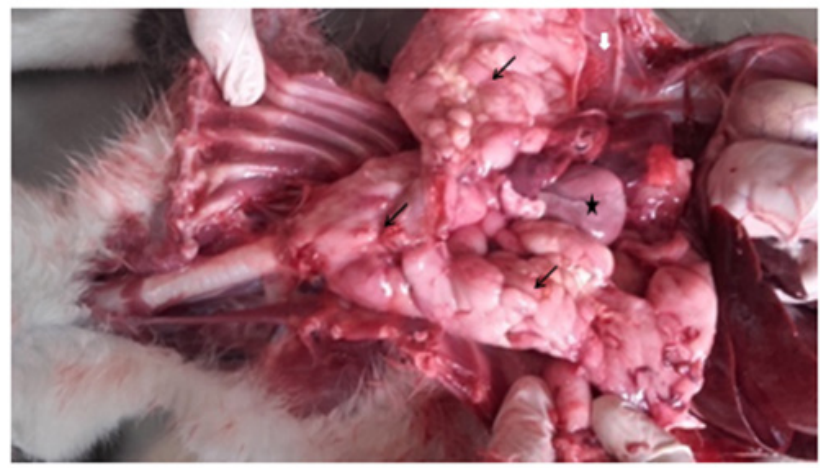

Figure 2 Image of the necropsy showing the important tumor mass (arrows), surrounding the heart (black star). Lung: white filled arrow. 


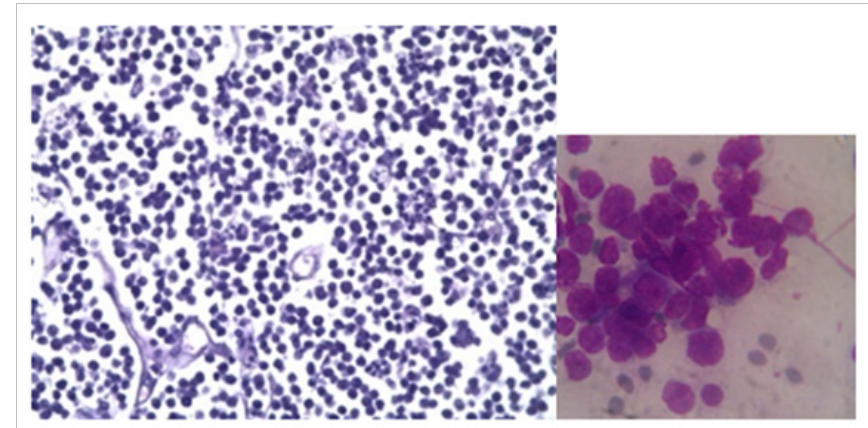

Figure 3 Histopathology: There is a proliferation of small lymphocytic cells (A, I0x). These are mainly circular and with little cytoplasm. The nuclei are round to elliptical and with some notches $(\mathrm{B}, 40 \mathrm{x})$. Presence of necrosis in the tumor tissue.

\section{Discussion}

According to several authors, the lymphoma has a bimodal clinical presentation pattern, the first group includes two-year-old cats, associated with ViLeF, compromising the mediastinal lymph nodes with the presence of pleural effusion. Due to the short age of the patients in this group, in addition to the lymph nodes, it can also affect the thymic tissue in the cranial mediastinum. The second group includes older cats, between 6 and 12 years old, not associated with ViLeF, in which the lymphoma is digestive or has a multicentre or extranodal pattern, the clinical signs depend on the affected organ. ${ }^{1-3}$

The patient presented some of the clinical signs and physical findings described in the literature for cats of the first group of the bimodal clinical presentation: dyspnea or tachypnea of acute presentation, associated with tumor size and pleural effusion, dysphagia, regional lymph node enlargement and attenuation of the cranial respiratory sounds. ${ }^{3}$ Other symptoms that are observed less frequently are: anorexia, weight loss, cough and ptyalism. In addition he presented positive serology for ViLeF. The serological determination of VIF and ViLeF is useful to determine the treatment of the cat with lymphoma, because the prognosis is reserved in the presence of retroviruses as a result of the weakness that it usually causes. ${ }^{4}$

In countries where vaccination is frequent, the association of this tumor with ViLeF-positive animals has been reduced to $25 \%$ compared to $60-70 \%$ of the cases published before the availability of the vaccine. ${ }^{2}$ Uruguay does not have ViLeF vaccine in the market, so the prevalence of this viral disease is high.

Although in the clinical case described, there was no immune reaction against the feline immunodeficiency virus (FIV), it is shown that it also increases the risk of lymphoma in the cat through indirect oncogenesis. ${ }^{2}$ Cats coinfected by ViLeF and FIV multiply 75 times the probability of developing a lymphoma. ${ }^{1,3}$
The results of the blood count (mild leukocytosis with a predominance of neutrophils) and serum biochemistry were nonspecific, as mentioned in the bibliography. ${ }^{5}$ Regenerative or regenerative anemia, neutrophilic leukocytosis, lymphocytosis, thrombocytopenia, hypoalbuminemia, hyperglobulinemia, hypercalcemia, prerenal or renal azotemia may also be observed, depending on the primary anatomic site and the paraneoplastic effects. ${ }^{5}$

Although it is described that the diagnosis can be established in $70-75 \%$ of the cats by fine needle aspiration and cytology of the cranial mediastinal mass (echo guided technique), in this case it was not performed since given the severity of dyspnea, humanitarian euthanasia was indicated. Histopathology aims at the differentiation between lymphoma and thymoma and performs staging to determine the prognosis of the disease. ${ }^{6}$

Other differentials for mediastinal masses that must be considered include heart-based tumor, ectopic thyroid or parathyroid tumor, thymic branchial cyst, chemo-dectoma (aortic or carotid body tumors), metastatic neoplasia, granuloma, abscess, lipoma, teratoma, and schwannoma.

\section{Acknowledgments}

None.

\section{Conflicts of interest}

The authors declare there is no conflict of interest.

\section{References}

1. Little SE. The cat: Clinical medicine and management. Canadá, Elsevier; 2011:1424.

2. Fabricio F, Calam AE, Dobsom JM, et al. Feline mediastinal lymphoma: a retrospective study of signalment, retroviral status, response to chemotherapy and prognostic indicators. J Feline Med Surg. 2014;16(8):637-644.

3. Nelson RW, Couto CG. Small animal internal medicine. 5th ed. Mosby, Elsevier; 2013:1504.

4. Smith AN. Cranial Mediastinal Masses: Diagnosis \& Therapy. 21 ST Annual American College of Veterinary Internal Medicine. Conference; 2003.

5. https://www.casadellibro.com/libro-consultas-en-medicina-internafelina/9789505551477/543890

6. Fidel LJ, Pargass IS, Dark MJ, et al. Granulocytopenia associated with thymoma in a domestic shorthaired cat. J Am Anim Hosp Assoc. 2008;44(4):210-217.

7. Zitz JC, Birchard SJ, Couto GC, et al. Results of excision of thymoma in cats and dogs: 20 cases (1984-2005). J Am Vet Med Assoc. 2008;232(8):1186-1192. 